\title{
Formation of power-law scalings of spectra and multi-scale coherent structures in the near-field of grid-generated turbulence
}

\author{
Tatsuya Yasuda ${ }^{*}{ }^{*}$ Susumu Goto ${ }^{2}{ }^{\dagger}$ and John Christos Vassilicos ${ }^{1 \ddagger}$ \\ 1 Turbulence, Mixing and Flow Control Group, \\ Department of Aeronautics, Imperial College London, United Kingdom and \\ 2 Graduate School of Engineering Science, Osaka University, Japan
}

(Dated: December 9, 2019)

\begin{abstract}
We investigate the streamwise evolutions of energy and pressure spectra along the shear-layer region of very near-field grid-generated turbulence. The energy and pressure spectra evolve significantly in this near field. The shear-layer and vortex-shedding frequencies appear immediately in different spectra but the shear-layer's spectral signature is very soon replaced by a broad power-law spectrum on both sides of that frequency. The spectra evolve further by filling the gap between the vortex shedding and the shear-layer frequencies eventually leading to near $-5 / 3$ power-law energy spectra at the point on the shear-layer region where the turbulence intensity reaches a maximum. The pressure spectrum reaches a $-7 / 3$ power-law shape significantly further downstream. These spectral scalings cover a range between the vortex-shedding frequency and frequencies larger than the shear-layer frequency. They are discussed in relation to turbulent coherent structures of various sizes, obtained by using Gaussian low-pass filtering of instantaneous turbulent flow fields. High enstrophy small-scale structures originate from the shear-layer instability whereas low enstrophy large-scale structures originate from the vortex shedding. The generation of near-field $-5 / 3$ energy and $-7 / 3$ pressure spectra involves cooperative interactions between these two different size structures.
\end{abstract}

\footnotetext{
* Current address: Department of Physical Science and Engineering, Nagoya Institute of Technology, Japan; yasuda.tatsuya@nitech.ac.jp

† goto@me.es.osaka-u.ac.jp

$\ddagger$ Current address: Univ. Lille, CNRS, ONERA, Arts et Métiers ParisTech, Centrale Lille, FRE 2017 LMFL - Laboratoire de Mécanique des fluides de Lille - Kampé de Feriet, F-59000 Lille, France; johnchristos.vassilicos@centralelille.fr
} 


\section{INTRODUCTION}

In laboratory and numerical experiments, we often observe the $-5 / 3$ power-law scaling of the energy spectrum $\widehat{E}(k)$ and the frequency power spectrum $E(f)$ of turbulence. For statistically homogeneous turbulence, the energy spectrum $\widehat{E}(k)$ and the pressure fluctuation

spectrum $\widehat{E}_{p}(k)$ are defined in terms of the Fourier transform of velocity and pressure fluctuations. One can show that these spectra are proportional to $k^{-5 / 3}$ and $k^{-7 / 3}$, respectively, by using a dimensional analysis $[1,2]$ based on the average of the energy dissipation rate $\bar{\epsilon}$ and the wavenumber $k$, which have been confirmed by experiments $[3,4]$ and numerical simulations [5]. If turbulence is statistically stationary and homogeneous, this dimensional analysis may be justified by the concept of the energy cascade; namely, the scale-by-scale energy transfer from larger to smaller scales ensures that the energy flux is independent of $k$ and is balanced by $\bar{\epsilon}$.

We emphasize, however, that this Richardson-Kolmogorov energy cascade picture assumes statistical homogeneity and stationarity. Since the energy cascade takes a finite time, when the turbulence is unsteady, the energy flux cannot be balanced by the energy dissipation rate. This implies that Kolmogorov's local equilibrium hypothesis does not hold for statistically non-stationary turbulence, even if it is statistically homogeneous (e.g. decaying turbulence in a periodic cube). In other words, although we sometimes observe $-5 / 3$ powerlaw scaling of the energy spectrum $\widehat{E}(k, t)$ in decaying turbulence, its origin remains not fully explained. Incidentally, even in statistically stationary turbulence, large-scale motions can show significant unsteadiness $[6,7]$.

The target of the present study is the turbulence which is statistically stationary but inhomogeneous. For this kind of turbulence (e.g., the near-field turbulence generated behind a grid in a wind tunnel), we can define the frequency power spectrum $E(f, \boldsymbol{x})$ but it depends on the location $\boldsymbol{x}$. Interestingly, the $-5 / 3$ power-law scaling of the frequency energy spectrum has been observed both experimentally and numerically, even in statistically inhomogeneous turbulent flows. For examples, Laizet et al. [8] and Gomes-Fernandes et al. [9] found a decade of $-5 / 3$ power-law scaling of frequency energy spectrum in a very nearfield region, where the flow is still statistically inhomogeneous, of a turbulent flow generated by a fractal square grid. Furthermore, in a turbulent flow generated by a single square element of a fractal square grid, Paul et al. [10] found a $-5 / 3$ slope in the frequency energy 


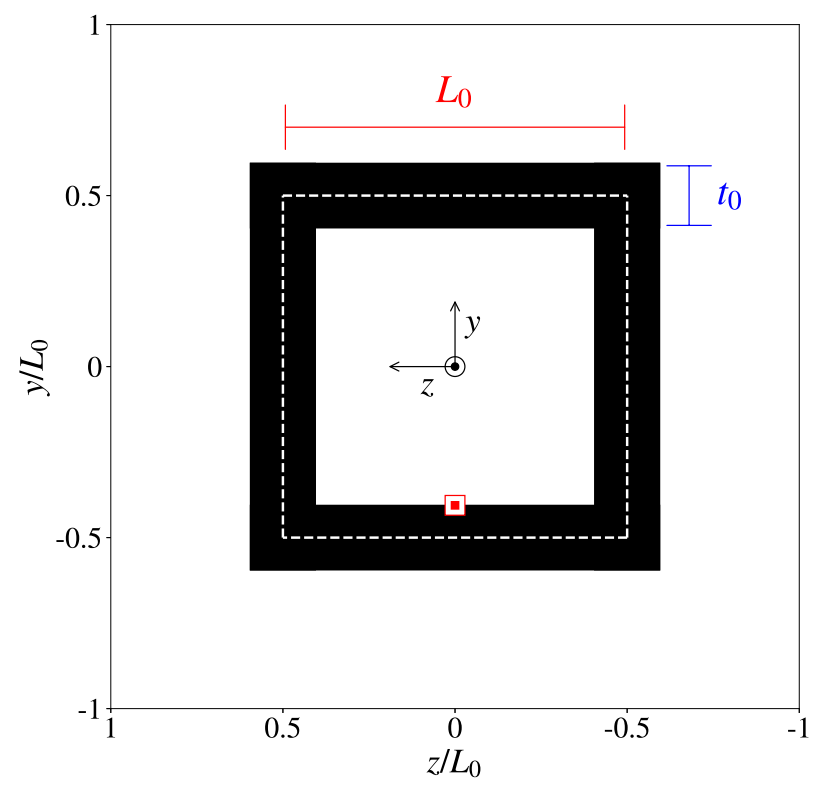

FIG. 1. Schematic of the grid used in this study. The length $L_{0}$ and the thickness $t_{0}$ are defined in the figure. This single square grid is placed on the inlet plane $x=0$ of the numerical domain of size $L_{x} \times L_{y} \times L_{z}=8 L_{0} \times 2 L_{0} \times 2 L_{0}$. The red-double square denotes the starting point of the shear-layer line shown in Fig. 2.

spectrum for half a decade of frequencies, despite the value of $R e_{\lambda}$ being approximately 20 . It is important to emphasize that finding a well-defined $-5 / 3$ frequency energy spectrum in the very near field is not limited to grid-generated turbulence but also other kinds of turbulent flows such as axisymmetric turbulent wakes [11] and wakes of a square prism [12]. These reports motivate us to tackle the following questions: (i) how are power-law scalings of energy spectra established in the very near field of grid-generated turbulence, i.e. highly inhomogeneous turbulence? (ii) does the Kolmogorov scaling of the pressure spectrum develop together with the energy spectrum? (iii) what kind of flow structures are relevant to realizing such power-law scalings?

To this end, we numerically investigate a turbulent flow generated with a single square grid [see Fig. 1] and a constant inflow velocity [10, 13-17], using Direct Numerical Simulation (DNS). In the previous studies, the presence of the power-law scalings of energy spectra and second-order velocity structure functions has been investigated along the centerline of the grid and along the bar-centerline. Our interest is to understand the origin of powerlaw scalings of energy and pressure spectra in the near field of the grid where the flow is 
statistically inhomogeneous. For this purpose, we have conducted long-time DNS so that we can investigate well-defined frequency spectra along a shear-layer line which we define in Sec. III.

The origin of multi-scale fluctuations related to power-law scalings of energy spectra is also discussed in this paper in terms of coherent structures across various length scales. We therefore use a coarse-graining method to filter specific length-scale fluctuations from continuous signals/fields of turbulence [e.g., 6, 7, 18-22]. The visualization method based on enstrophy isosurfaces can only detect small-scale dissipative structures, whereas our aim is to detect coherent vortical structures at various scales. Such methods have been exploited for several turbulent flows [7, 19, 22-24]. For example, Goto et al. [7] and Motoori and Goto [22] have demonstrated a hierarchy of tubular vortical structures of different sizes in the inertial subrange in triply periodic turbulence (see e.g. Fig. 1 in Ref. [7]) and in a turbulent boundary layer (see e.g. Fig. 4 in Ref. [22]). This approach is effective when investigating multi-scale spatial fluctuations relevant for power-law scalings of spectra in our grid-generated turbulence.

Our aim in this paper is to investigate the spatial origin of power-law scalings in energy and pressure spectra and multi-scale coherent structures related to them in the inhomogeneous and anisotropic near field of grid-generated turbulence. This paper is composed as follows. In the next section, we explain the numerical methods, conditions, and our analysis tools. In Sec. III, we firstly examine the streamwise evolution of turbulence statistics along the shear-layer line. Secondly, we pay some special attention to two characteristic frequencies detected in the grid-generated turbulence. Thirdly, we present evidence of turbulent coherent structures at several scales in the grid-generated turbulence, by exploiting three-dimensional flow field data of DNS. To the best of our knowledge, this is the first such attempt in the grid-generated turbulence. Fourthly, we discuss inter-scale energy fluxes in relation to the other results of this section. Finally, we summarize our conclusions in Sec. IV. 


\section{DIRECT NUMERICAL SIMULATIONS}

\section{A. Numerical schemes and parameters}

We perform DNS of single-square grid-generated turbulence by solving the momentum equation,

$$
\frac{\partial \boldsymbol{u}}{\partial t}+\boldsymbol{u} \cdot \nabla \boldsymbol{u}=-\frac{1}{\rho} \nabla p+\nu \nabla^{2} \boldsymbol{u}+\boldsymbol{f}
$$

with the continuity equation,

$$
\nabla \cdot \boldsymbol{u}=0
$$

where $\boldsymbol{u}$ is the velocity field, $\rho$ and $\nu$ are, respectively, the density and the kinematic viscosity of the fluid, both of which are assumed to be constant, and $\boldsymbol{f}$ is the external force used in an immersed boundary method. As shown in Fig. 1, $x$ is the streamwise direction and $y$ and $z$ are the two-cross flow directions. The periodic boundary conditions are imposed for $y$ - and $z$-directions. The inflow velocity at the beginning of the computational domain in the streamwise direction is $U_{\infty}$, and no disturbance is imposed on the inlet flow. For the outlet flow at $x=L_{x}$, a standard convection equation is used.

We use a flow solver, Incompact3d [25, 26], to integrate (1) and (2), which can be run on parallel supercomputers using a two-dimensional domain decomposition strategy. The solver is based on sixth order compact finite schemes [27] for spatial differentiation and the thirdorder Adams-Bashforth scheme for time integration. In order to treat the incompressibility condition (2), a fractional step method is employed and the Poisson equation is solved in Fourier space. More details about the solver are given in Refs. [25, 26].

The grid is modelled by using an immersed boundary method [28]. This method enables, by adding an extra forcing $\boldsymbol{f}$ in (1), to enforce the no-slip boundary condition on the wall of the grid. The shape of the grid used is shown in Fig. 1. $L_{0}$ is the lateral length of each bar of the grid and $t_{0}$ is their lateral thickness, where $L_{0}=5.3 t_{0}$, and their streamwise thickness is $0.25 t_{0}$. The computational domain is $L_{x} \times L_{y} \times L_{z}=8 L_{0} \times 2 L_{0} \times 2 L_{0}$ and is descritized with $2881 \times 720 \times 720$ grid points. Our parameter setting is the same as the one used for the highest resolution DNS of Laizet et al. [29]. The spatial resolution $\Delta=\Delta_{x}=\Delta_{y}=\Delta_{z}$ lies between $0.5\langle\eta\rangle$ and $2\langle\eta\rangle$ throughout the domain, where $\eta=\nu^{\frac{3}{4}} \epsilon^{-\frac{1}{4}}$ is the Kolmogorov length scale and the brackets $\langle\cdot\rangle$ denote the time average. More details on 
the validation of the simulation can be found in Ref. [29], where the resolution effects on finescale features and in particular on vorticity and strain rate statistics using the $Q-R$ diagram [30] are demonstrated. We have run a set of simulations for $6.94 \leq t /\left(L_{0} / U_{\infty}\right) \leq 55.56$, so that the total time length of our simulation is $48.61 L_{0} / U_{\infty}$. The initial condition of our simulation is taken from fully developed turbulent flow. We have stored the numerical data of 17,500 snapshots of velocity and pressure fields in the subdomain $\left(0 \leq x / x_{*} \leq 0.315\right.$, $\left.-0.75 \leq y / L_{0} \leq 0.25,-0.25 \leq z / L_{0} \leq 0.25\right)$, with a time interval, $2.8 \times 10^{-3} L_{0} / U_{\infty}$. We emphasize that this sufficiently long time series data enables us to precisely estimate time averages (denoted by brackets $\langle\cdot\rangle$ ) and power spectra of the three velocity components and pressure. We have also stored the numerical data of fewer snapshots of velocity and pressure fields in the full computational domain which are used for flow visualizations (see, e.g. Fig. 9).

In the present study, the streamwise distance $x$ from the grid is non-dimensionalized using the wake interaction length-scale [31, 32],

$$
x_{*}=L_{0}^{2} / t_{0},
$$

and the full computational domain is set to $-0.236 \leq x / x_{*} \leq 1.27,-1 \leq y / L_{0}, z / L_{0} \leq 1$. Our global Reynolds numbers $R e_{L_{0}}=L_{0} U_{\infty} / \nu$ and $R e_{t_{0}}=t_{0} U_{\infty} / \nu$ are 21600 and 4075, respectively. The Reynolds number $R e_{t_{0}}=4075$ lies in the range $(R e>1300)$ where, for circular cylinder wakes, Kelvin-Helmholtz vortices can be generated in the shear layer past of the bluff body. (See Ref. [33].)

We also introduce the non-dimensional frequency,

$$
\mathrm{St}=f t_{0} / U_{\infty}
$$

based on the bar thickness $t_{0}$. In our setup, the vortex shedding Strouhal number $\mathrm{St}_{s}$ (defined in terms of $t_{0}$ and $U_{\infty}$ ) is found to be 0.186 . This value of the shedding Strouhal number is in good agreement with those of experiments. Melina et al. [16] determinded experimentally that the vortex shedding Strouhal number of grid-generated turbulence with a single square grid $\left(L_{0}=229.0 \mathrm{~mm}\right.$ and $\left.t_{0}=43 \mathrm{~mm}\right)$ as the one investigated in this study is about 0.19 for two different inlet velocities $\left(U_{\infty}=5\right.$ and $\left.17 \mathrm{~m} / \mathrm{s}\right)$. Their Taylor scale-based Reynolds number at $x / x_{*} \approx 0.5$ on the centerline is $R e_{\lambda} \approx 200$ for $U_{\infty}=5 \mathrm{~m} / \mathrm{s}$ and $R e_{\lambda} \approx 350$ for $U_{\infty}=17 \mathrm{~m} / \mathrm{s}$, while our simulation returns $R e_{\lambda} \approx 100$ at $x / x_{*}=0.5$. Numerical results 
for the streamwise evolution of $R e_{\lambda}$ along the centerline have been reported in Fig. 7 of Ref. [29].

\section{B. Coarse-graining method}

Turbulent flows are composed of coherent structures [34, 35]. Topologies of fine-scale coherent structures have been investigated in terms of invariants of the velocity gradient tensor [8, 36-41]. One may decompose the velocity gradient tensor into the rate of strain

tensor $s_{i j}=\frac{1}{2}\left(\frac{\partial u_{j}}{\partial x_{i}}+\frac{\partial u_{i}}{\partial x_{j}}\right)$ and the rate of rotation tensor $\omega_{i j}=\frac{1}{2}\left(\frac{\partial u_{j}}{\partial x_{i}}-\frac{\partial u_{i}}{\partial x_{j}}\right)$. The second invariant $Q$ of the velocity gradient tensor can be written as

$$
Q=Q_{\omega}+Q_{s}
$$

where

$$
Q_{\omega}=\frac{1}{2} \omega_{i j} \omega_{i j} \quad \text { and } \quad Q_{s}=-\frac{1}{2} s_{i j} s_{i j}
$$

Locations in the flow where $Q>0$ are dominated by vorticity rather than strain. This is one of the quantities exploited to visualize fine-scale coherent motions in a variety of flow geometries [37, 38].

Since the velocity gradient is predominately determined by dissipation-scale motions, flow visualizations of physical quantities based on velocity gradients, by nature, highlight dissipative small-scale coherent structures in the flow. Our interest lies in multi-scale flow structures in the flow relating to the broad spectra which will be shown in Sec. III B. Therefore, in this study we apply coarse-graining to instantaneous turbulent flow fields [6, 7, 18-21] in order to extract spatial fluctuations with several length scales.

In the present study, we use the three-dimensional Gaussian low-pass filter, which is written in physical space as

$$
G(\boldsymbol{r} ; \sigma)=\frac{1}{(\sqrt{2 \pi} \sigma)^{3}} e^{-\frac{|\boldsymbol{r}|^{2}}{2 \sigma^{2}}} .
$$

Here, $\sigma$ is the filter width. By using this filter, a quantity $A(\boldsymbol{x})$ is coarse-grained as follows:

$$
\widetilde{A}(\boldsymbol{x} ; \sigma)=\int G\left(\boldsymbol{x}-\boldsymbol{x}^{\prime} ; \sigma\right) A\left(\boldsymbol{x}^{\prime}\right) d \boldsymbol{x}^{\prime} .
$$

It is sometimes useful to do the coarse-graining in Fourier space because the Fourier transform of $\widetilde{A}(\boldsymbol{x} ; \sigma)$ is expressed by

$$
\widehat{\widetilde{A}}\left(\boldsymbol{k} ; k_{c}\right)=\widehat{G}\left(\boldsymbol{k} ; k_{c}\right) \widehat{A}(\boldsymbol{k})
$$


with

$$
\widehat{G}\left(\boldsymbol{k} ; k_{c}\right)=e^{-\frac{|\boldsymbol{k}|^{2}}{2 k_{c}^{2}}},
$$

where $k_{c}=1 / \sigma$ is the cut-off wavenumber. We apply Gaussian filtering in either real space (8) or Fourier space (9) depending on the data structure. Gaussian filters were used in previous studies to investigate multi-scale structures in triply-periodic turbulence [19] and wall-bounded turbulence [22-24].

It is useful to connect spatial with temporal variations of velocity signals. For this, we need to relate the cut-off wavenumber $k_{c}$ of the Gaussian filter (10) to a frequency $f$. Guided by the concept encapsulated in Taylor's frozen hypothesis [42, 43], we define the cut-off wavenumber,

$$
k_{c}(\mathrm{St})=2 \pi f / U_{\infty}=2 \pi \mathrm{St} / t_{0},
$$

in terms of the inflow velocity $U_{\infty}$, so that $k_{c}$ is a function of non-dimensional frequency St $\left(=f t_{0} / U_{\infty}\right)$. This cut-off wavenumber $k_{c}$ is used in Sec. III C to make a link with frequency spectra, which are shown in Sec. III B.

Finally, we define the coarse-grained quantities used in the present study. After obtaining the coarse-grained velocity gradient tensor $\frac{\partial \widetilde{u}_{j}}{\partial x_{i}}$ with the use of Eq. (8) or Eq. (9), we decompose it into the coarse-grained strain-rate tensor $\widetilde{s}_{i j}=\frac{1}{2}\left(\frac{\partial \widetilde{u}_{j}}{\partial x_{i}}+\frac{\partial \widetilde{u}_{i}}{\partial x_{j}}\right)$ and the coarse-grained rotation-rate tensor $\widetilde{\omega}_{i j}=\frac{1}{2}\left(\frac{\partial \widetilde{u}_{j}}{\partial x_{i}}-\frac{\partial \widetilde{u}_{i}}{\partial x_{j}}\right)$. Using these, we define the second invariant $\widetilde{Q}$ of the coarse-grained velocity gradient tensor, as

$$
\widetilde{Q}=\widetilde{Q}_{\omega}+\widetilde{Q}_{s}
$$

where

$$
\widetilde{Q}_{\omega}=\frac{1}{2} \widetilde{\omega}_{i j} \widetilde{\omega}_{i j} \quad \text { and } \quad \widetilde{Q}_{s}=-\frac{1}{2} \widetilde{s}_{i j} \widetilde{s}_{i j}
$$

\section{RESULTS}

\section{A. Streamwise evolution of energy and pressure fluctuations}

In grid-generated turbulence, there exist shear-layer regions past the bars of the grid. The Reynolds number $R e_{t_{0}}$ based on $t_{0}$ of our simulation is 4075 and therefore lies in the range $(R e>1300)$ where Kelvin-Helmholtz vortices can be generated in the shear-layer 


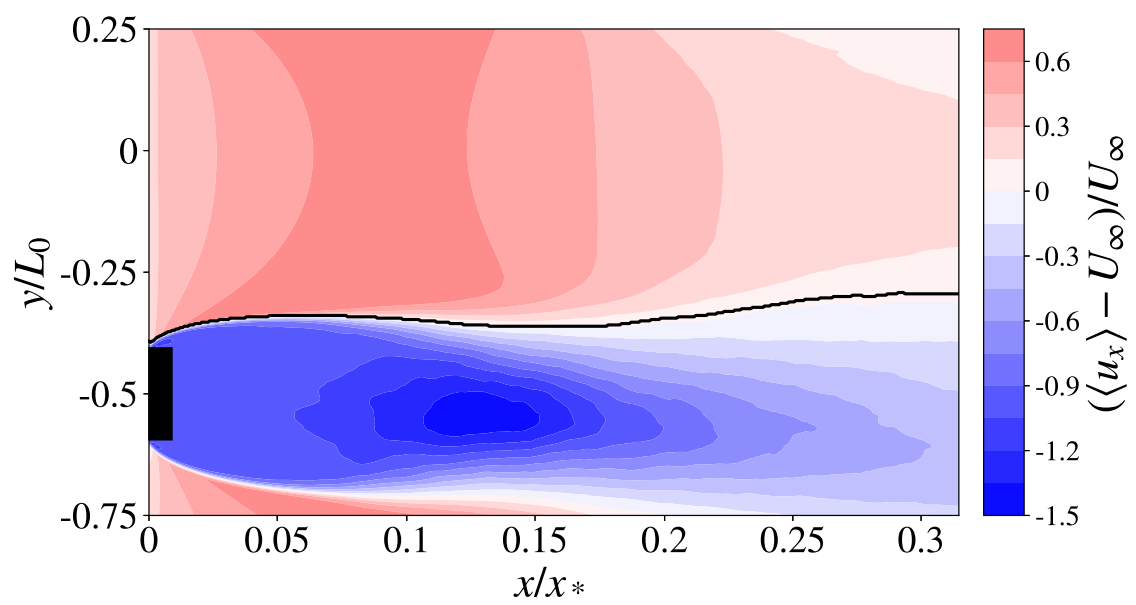

FIG. 2. The mean field of streamwise velocity obtained by subtracting the inflow velocity $U_{\infty}$ on the plane at $z=0$. The time average is taken over the period $6.94 \leq t /\left(L_{0} / U_{\infty}\right) \leq 55.56$. The black line denotes the locations which satisfy $\left\langle u_{x}\right\rangle-U_{\infty}=0$. The cross-section of the grid shown in Fig. 1 is illustrated by the black rectangle.

regions past a circular cylinder (see Ref. [33]). Similarly to this observation in the wake of a circular cylinder, intense turbulent fluctuations start to be generated in shear layers behind the bars of the grid shown in Fig. 1. Let us first demonstrate how the signatures of developed turbulence such as the $-5 / 3$ power-law scaling of energy spectra appear and develop along a shear layer. For this purpose, we define the shear-layer line by the locations $\boldsymbol{x}$ past a bar of the grid which satisfy

$$
\left\langle u_{x}\right\rangle(\boldsymbol{x})-U_{\infty}=0
$$

In Fig. 2, the shear-layer line is represented by the black thick line, which separates $\left\langle u_{x}\right\rangle>$ $U_{\infty}$ from $\left\langle u_{x}\right\rangle<U_{\infty}$. The starting point of the shear-layer line is indicated by the red double square in Fig. 1, whose coordinate is $(x, y, z)=\left(0,-\left(L_{0}-t_{0}\right) / 2+0.0589 t_{0}, 0\right)$.

Figure 3 shows streamwise evolutions of turbulent intensities $I_{x}=\sqrt{\left\langle u_{x}^{\prime 2}\right\rangle} / U_{\infty}, I_{y}=$ $\sqrt{\left\langle u_{y}^{\prime 2}\right\rangle} / U_{\infty}$, and $I_{z}=\sqrt{\left\langle u_{z}^{\prime 2}\right\rangle} / U_{\infty}$ in $x$-, $y$-, and $z$-directions, and root-mean-square pressure fluctuation $p^{\mathrm{rms}}=\sqrt{\left\langle p^{\prime 2}\right\rangle} / \rho U_{\infty}^{2}$, along the shear-layer line, where $u_{x}^{\prime}=u_{x}-\left\langle u_{x}\right\rangle, u_{y}^{\prime}=$ $u_{y}-\left\langle u_{y}\right\rangle$, and $u_{z}^{\prime}=u_{z}-\left\langle u_{z}\right\rangle$ are the three fluctuating velocity components and $p^{\prime}=p-\langle p\rangle$ is the pressure fluctuation. The evolutions of the turbulent intensities are qualitatively similar to those previously found along the centerline of grid-generated turbulence $[9,31,32,44,45]$ and within shear layers in the near field of the turbulent flow past a square cylinder [41]. 


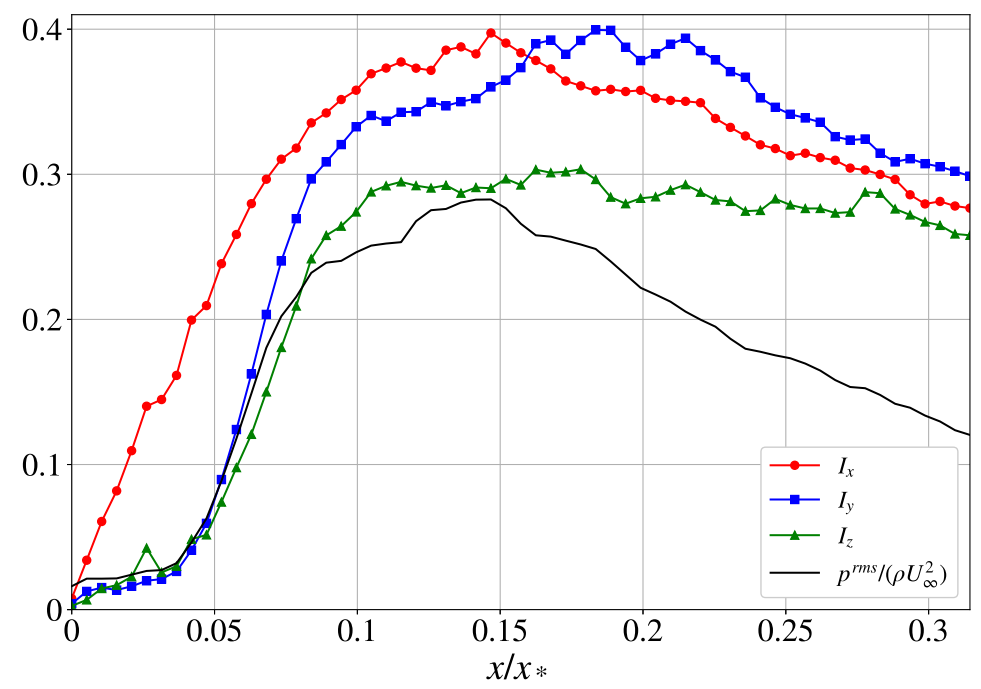

FIG. 3. The streamwise evolution of root mean square velocity components and pressure along the shear-layer line as a function of the streamwise coordinate. The time average is taken for the period $6.94 \leq t /\left(L_{0} / U_{\infty}\right) \leq 55.56$.

The peak of $I_{x}$ is found at $x / x_{*} \approx 0.15$. We hereinafter refer to $0 \leq x / x_{*} \lesssim 0.15$ as the production region and $0.15 \lesssim x / x_{*}$ as the decay region. $I_{x}$ is much larger than $I_{y}$ and $I_{z}$ until $x / x_{*}=0.15$ and $I_{y}$ becomes the largest beyond $x / x_{*}=0.15$. Evidently, turbulent velocity fluctuations are statistically anisotropic along the shear-layer line, particularly in the very near field $\left(0 \leq x / x_{*} \lesssim 0.05\right)$.

In Fig. 4 we plot the space-time diagrams which show the time evolutions $(48.6 \leq$ $\left.t /\left(L_{0} / U_{\infty}\right) \leq 51.6\right)$ of fluctuating velocity components $u_{x}^{\prime} / U_{\infty}, u_{y}^{\prime} / U_{\infty}, u_{z}^{\prime} / U_{\infty}$, and fluctuating pressure $p^{\prime} / \rho U_{\infty}^{2}$ along the shear-layer line. It is found in the diagrams that, going downstream from the starting point $x / x_{*}=0$, intense high-frequency fluctuations start to appear at $x / x_{*} \approx 0.05$ for all the quantities. Going further downstream $\left(0.15 \lesssim x / x_{*}\right)$, the frequency of alternations of positive-valued and negative-valued contours becomes lower. As shown below, the former high-frequency fluctuations stem from the shear-layer instability, whereas the latter from the vortex shedding. 

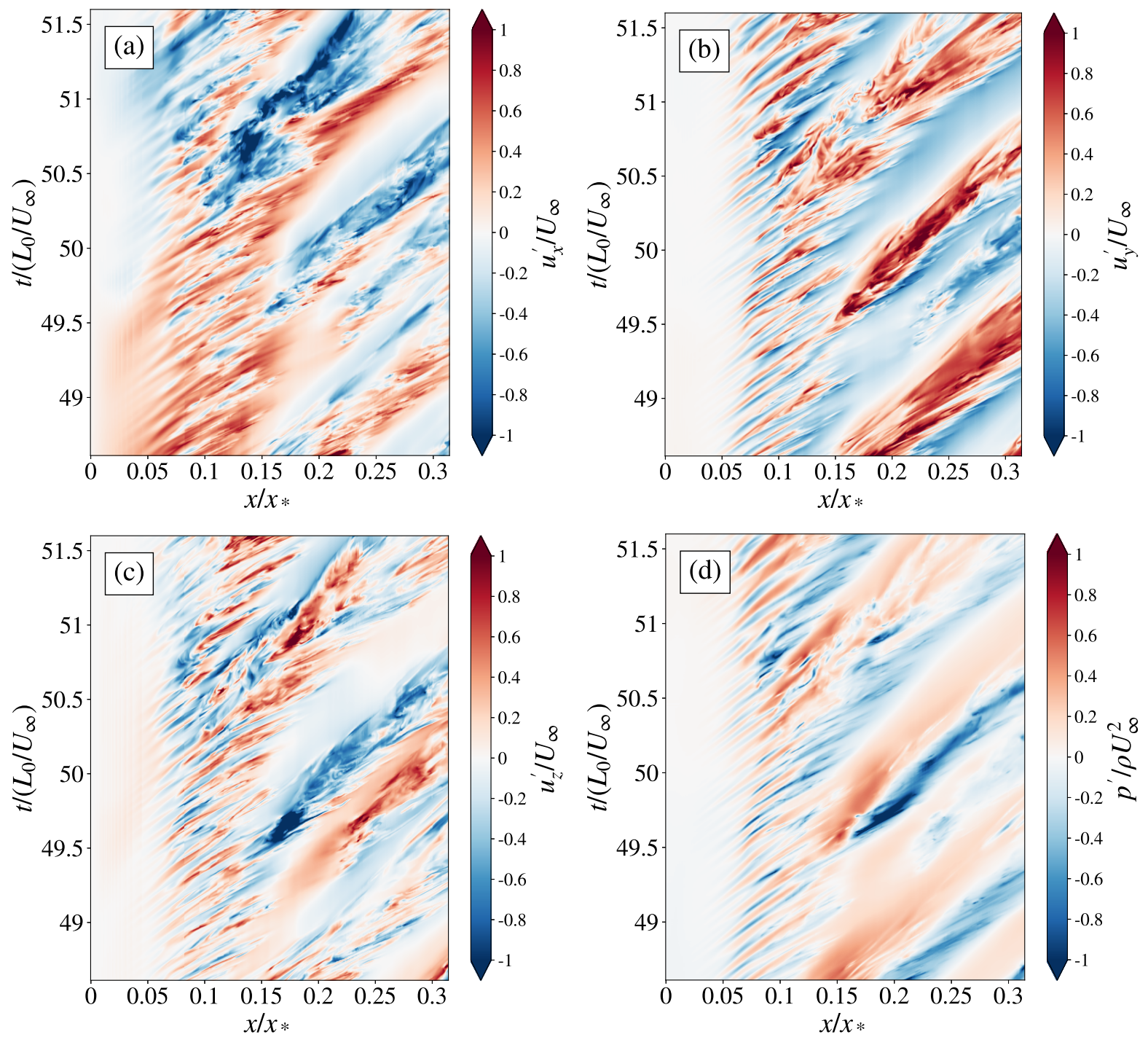

FIG. 4. Space-time diagrams of (a) $u_{x}^{\prime}$, (b) $u_{y}^{\prime}$, (c) $u_{z}^{\prime}$, and (d) $p^{\prime}$. The horizontal axis shows the streamwise coordinate $\left(0 \leq x / x_{*} \leq 0.315\right)$, and the vertical axis shows the time $(48.6 \leq$ $\left.t /\left(L_{0} / U_{\infty}\right) \leq 51.6\right)$

\section{B. Streamwise evolution of energy and pressure spectra}

We now move to the frequency spectra of the three fluctuating velocity components and the fluctuating pressure, which are defined, respectively, by

$$
E_{i}(f)=\lim _{T \rightarrow \infty} \frac{1}{T}\left|\widehat{u}_{i}^{\prime}(f)\right|^{2} \quad(i=x, y, z) \quad \text { and } \quad E_{p}(f)=\lim _{T \rightarrow \infty} \frac{1}{T}\left|\widehat{p}^{\prime}(f)\right|^{2}
$$



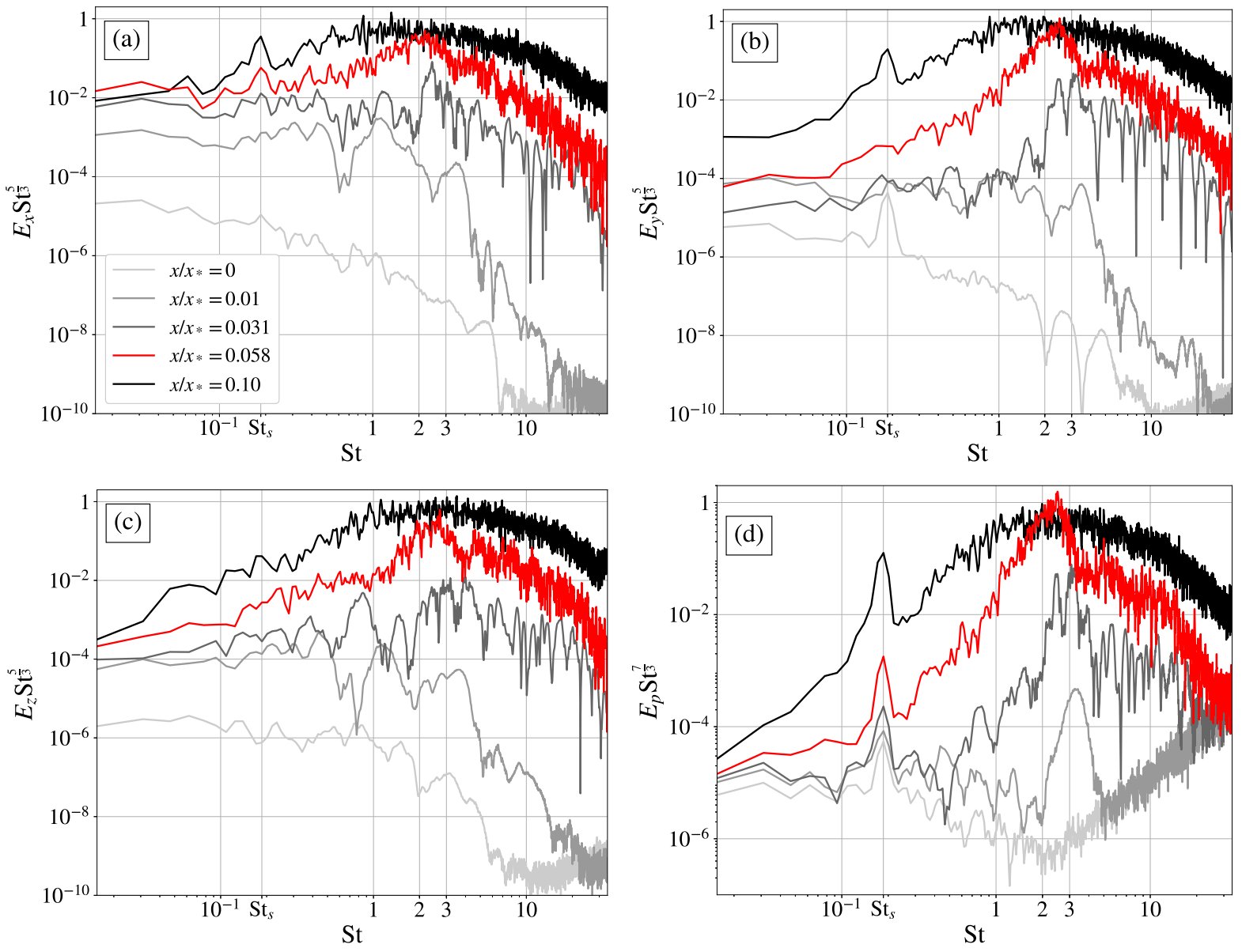

FIG. 5. Logarithmic plots of the compensated power spectra (a) $E_{x} \mathrm{St}^{\frac{5}{3}}$, (b) $E_{y} \mathrm{St}^{\frac{5}{3}}$, (c) $E_{z} \mathrm{St}^{\frac{5}{3}}$, and (d) $E_{p} \mathrm{St}^{\frac{7}{3}}$, against the Strouhal number $\mathrm{St}=f t_{0} / U_{\infty}$, at the six different locations in the shear-layer line: $x / x_{*}=0,0.01,0.031,0.058$, and 0.10 .

They are computed on the basis of the Fourier transforms $\widehat{u}_{x}^{\prime}, \widehat{u}_{y}^{\prime}, \widehat{u}_{z}^{\prime}$, and $\widehat{p^{\prime}}$ of long time series data for $u_{x}^{\prime}, u_{y}^{\prime}, u_{z}^{\prime}$ and $p^{\prime}\left(6.94 \leq t /\left(L_{0} / U_{\infty}\right) \leq 55.56\right)$. Figure 5 shows the compensated frequency spectra of velocity and pressure fluctuations at five different locations $x / x_{*}=0,0.01,0.031,0.058$, and 0.10 along the shear-layer line. The velocity and pressure spectra are multiplied by $\mathrm{St}^{\frac{5}{3}}$ and $\mathrm{St}^{\frac{7}{3}}$, respectively. It is clear that the spectra undergo a drastic change while moving downstream. The energy spectra seem to be developing a continuous region at $x / x_{*} \approx 0.1$.

Another observation regarding the spectra in the production region is that they have strong peaks at two characteristic frequencies: one is at $\mathrm{St}=0.186$ and the other is around $2 \lesssim$ St $\lesssim 3$. The lower frequency is the so-called vortex-shedding frequency [16]. The 

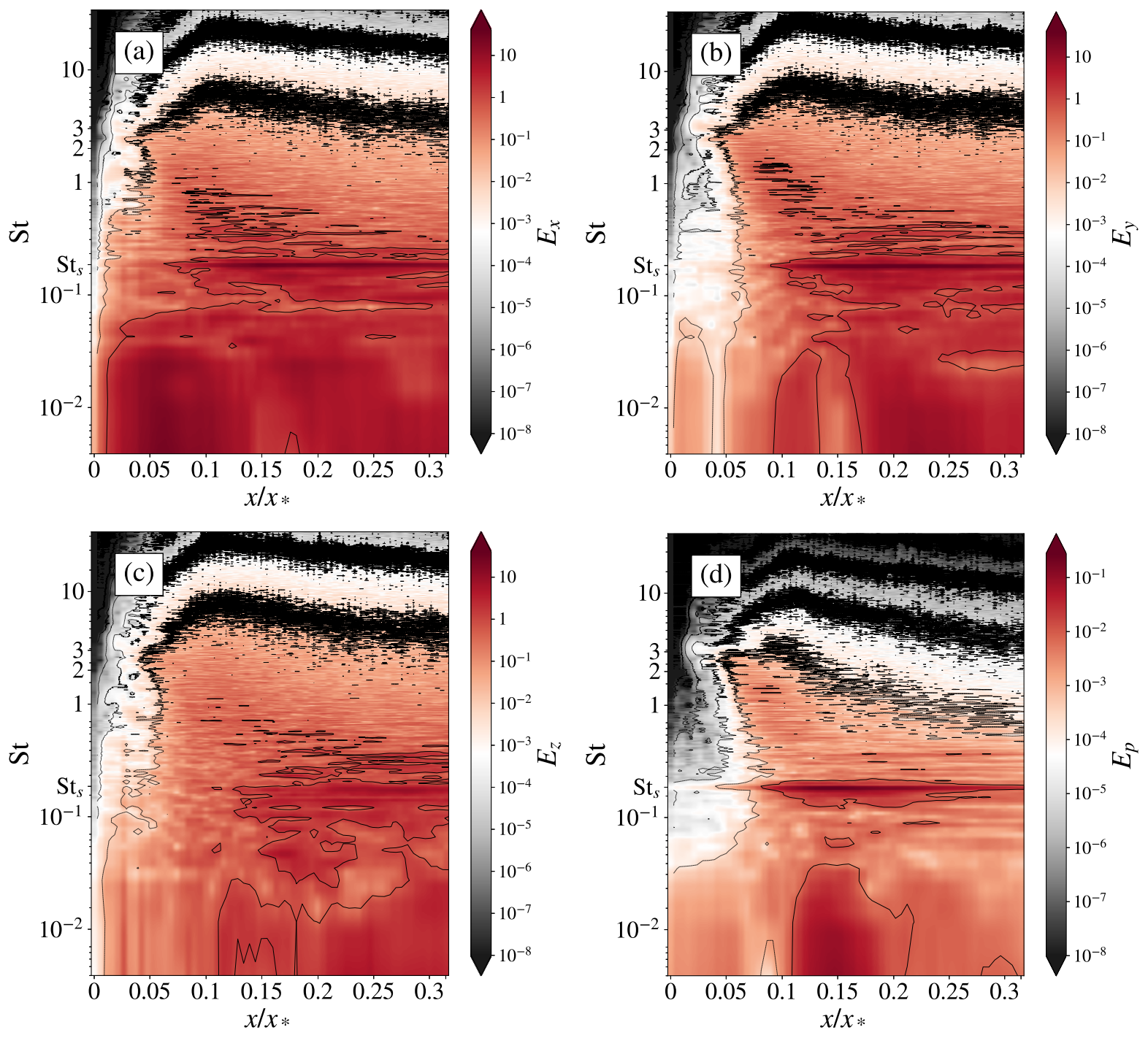

FIG. 6. Streamwise evolution of power spectra (a) $E_{x}$, (b) $E_{y}$, (c) $E_{z}$, and (d) $E_{p}$. The horizontal axis shows the streamwise coordinate $\left(0 \leq x / x_{*} \leq 0.315\right)$.

higher frequency is related to the shear-layer instability [46-48]. Around the streamwise location $x / x_{*} \approx 0.031$, energy and pressure spectra, especially $E_{y}(\mathrm{St})$ and $E_{p}(\mathrm{St})$, start to demonstrate a significant growth around $2 \lesssim \mathrm{St} \lesssim 3$ [see Figs. 5(b,d)]. This frequency is about 10 times larger than the vortex-shedding frequency. It is important to note that the spectra first acquire (see the spectra at $x / x_{*}=0.058$ ) the energy at the shear-layer instability frequency and the spectra around the vortex-shedding frequency grow later.

To identify the origin of these two characteristic peaks of the spectra, we show, in Fig. 6, plots of power spectra of velocities and pressure as functions of the streamwise location. It 
is clear in Figs. 6(a) and 6(b) that $E_{x}$ and $E_{y}$ at the vortex-shedding frequency $\mathrm{St}=0.186$ start to become intense at $x / x_{*} \approx 0.1$. Because the central axis of rotation of large-scale vortices shed in the shear layers is aligned parallel to $z$-axis, as seen in Fig. 10, the peak at the vortex-shedding frequency in $E_{z}$ is not high relative to those in $E_{x}$ and $E_{y}$ [compare Fig. 6(c) with Figs. 6(a) and 6(b)]. Crucially, the significant peak of $E_{p}$ at the vortex shedding frequency is observed throughout the streamwise range shown in Fig. 6(d).

Next, let us look at the evolution of the shape of the spectra in the downstream region, $0.058 \leq x / x_{*} \leq 0.28$ (Fig. 7). There is a clear tendency that, in the production region [see black lines in Figs. $7(\mathrm{a}, \mathrm{b}, \mathrm{c})]$, the energy spectra at the shear-layer frequency first become intense, and then those around the vortex-shedding frequency become stronger while those at the shear-layer frequency become weaker. As the spectra at the vortex-shedding frequency become weaker, the energy spectra gradually start to demonstrate a very defined $-5 / 3$ power-law scaling. The energy spectra in the decay region, on the other hand, keep the $-5 / 3$ power-law scaling [see blue lines in Figs. $7(\mathrm{a}, \mathrm{b}, \mathrm{c})$ ], where the peaks of $E_{x}$ and $E_{y}$ at the shedding frequency are persistent.

We emphasize that this spatial evolution of the energy spectrum along the shear-layer line is qualitatively different from the one along the centerline of the grid. This is seen by comparing the above observation (Fig. 7) with Fig. 20 in Ref. [8] and Fig. 8 in Ref. [9]. The first difference is that the shear-layer frequency is absent on the spectra along the centerline before a $-5 / 3$ power-law scaling emerges. The second difference is that along the shearlayer line the slope of the inertial range spectra is found to be steeper before $-5 / 3$ energy spectrum appears, whereas they are shallower along the centerline. Along the shear-layer line, as examined in the next subsection, turbulent fluctuations start to build up from smaller to larger scales.

Another intriguing observation relates to the streamwise evolution of the pressure spectrum. The way that the flow approaches the turbulent state characterized by the Kolmogorov-like scaling is different between the energy and pressure spectra in the decay region (see blue lines in Fig. 7). Beyond the location where turbulence intensities are maximal $\left(\approx 0.15 x_{*}\right)$, the energy spectra show the $-5 / 3$ power-law scaling and this scaling exponent remains the same up to $x / x_{*}=0.315$. For the pressure spectrum, on the other hand, the scaling exponent is decreasing along the downstream direction such that it is close to $-5 / 3$ at $x / x_{*}=0.16,-2$ at $x / x_{*}=0.2$, and $-7 / 3$ at $x / x_{*}=0.28$. This dissimilarity of 

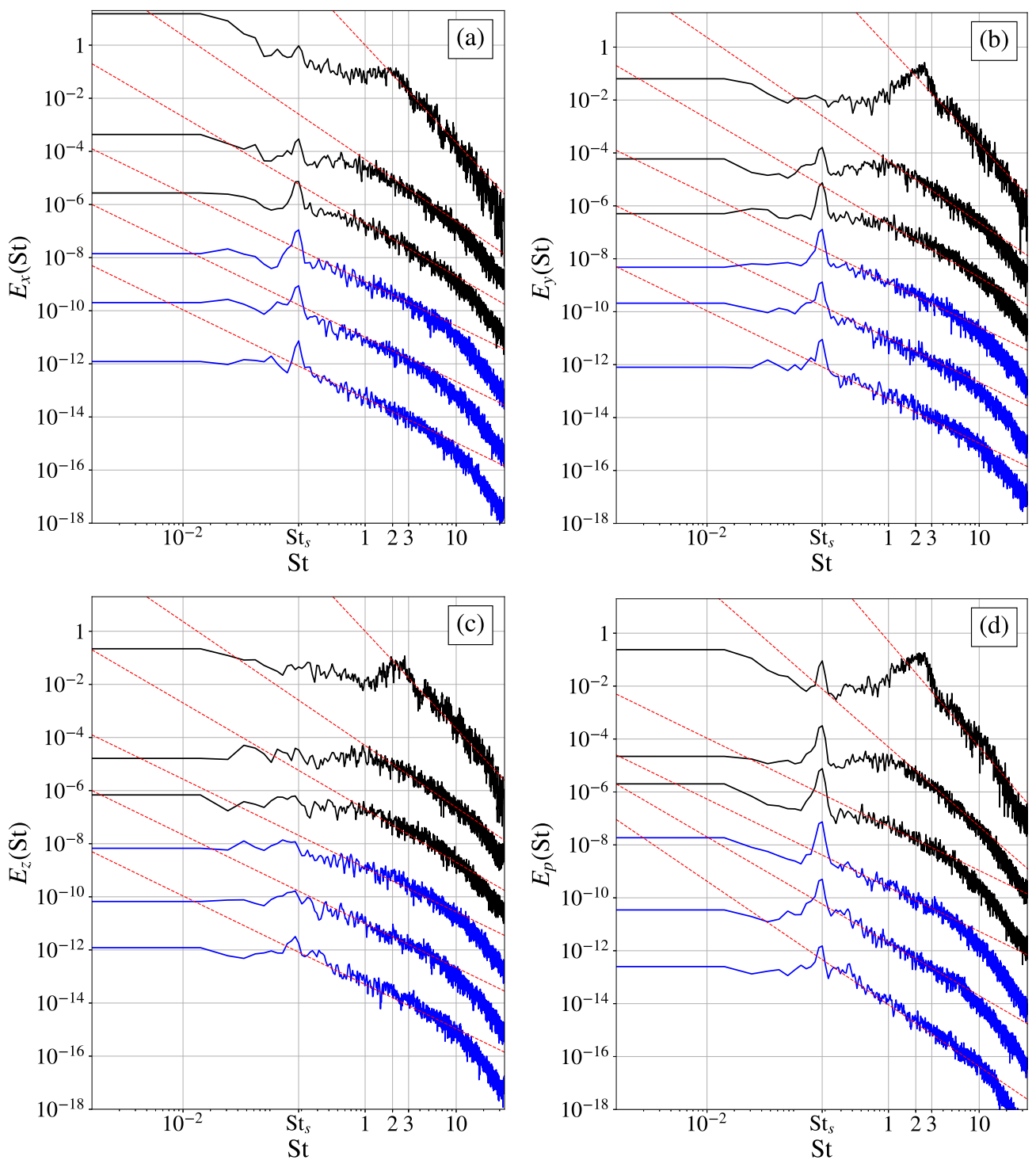

FIG. 7. Logarithmic plots of the power spectra (a) $E_{x}$, (b) $E_{y}$, (c) $E_{z}$, and (d) $E_{p}$, against the Strouhal number $\mathrm{St}=f t_{0} / U_{\infty}$. The spectra are computed at the six different streamwise locations in the shear-layer line: $x / x_{*}=0.058,0.1,0.13,0.16,0.2$, and 0.28 from top to bottom. For clarity, the spectra shown are shifted downward along the vertical axis by multiplying by a factor of $5.0 \times 10^{-5}, 5.0 \times 10^{-7}, 5.0 \times 10^{-9}, 5.0 \times 10^{-11}$ and $5.0 \times 10^{-13}$, with the exception of the ones on the top. The black-colored and blue-colored spectra belong to the production and decay regions, respectively. The red lines from top to bottom denote the slopes of power-law exponents (a,b,c) $-11 / 3,-7 / 3,-2,-5 / 3,-5 / 3,-5 / 3$ and $(\mathrm{d})-4,-3,-5 / 3,-5 / 3,-2,-7 / 3$. 

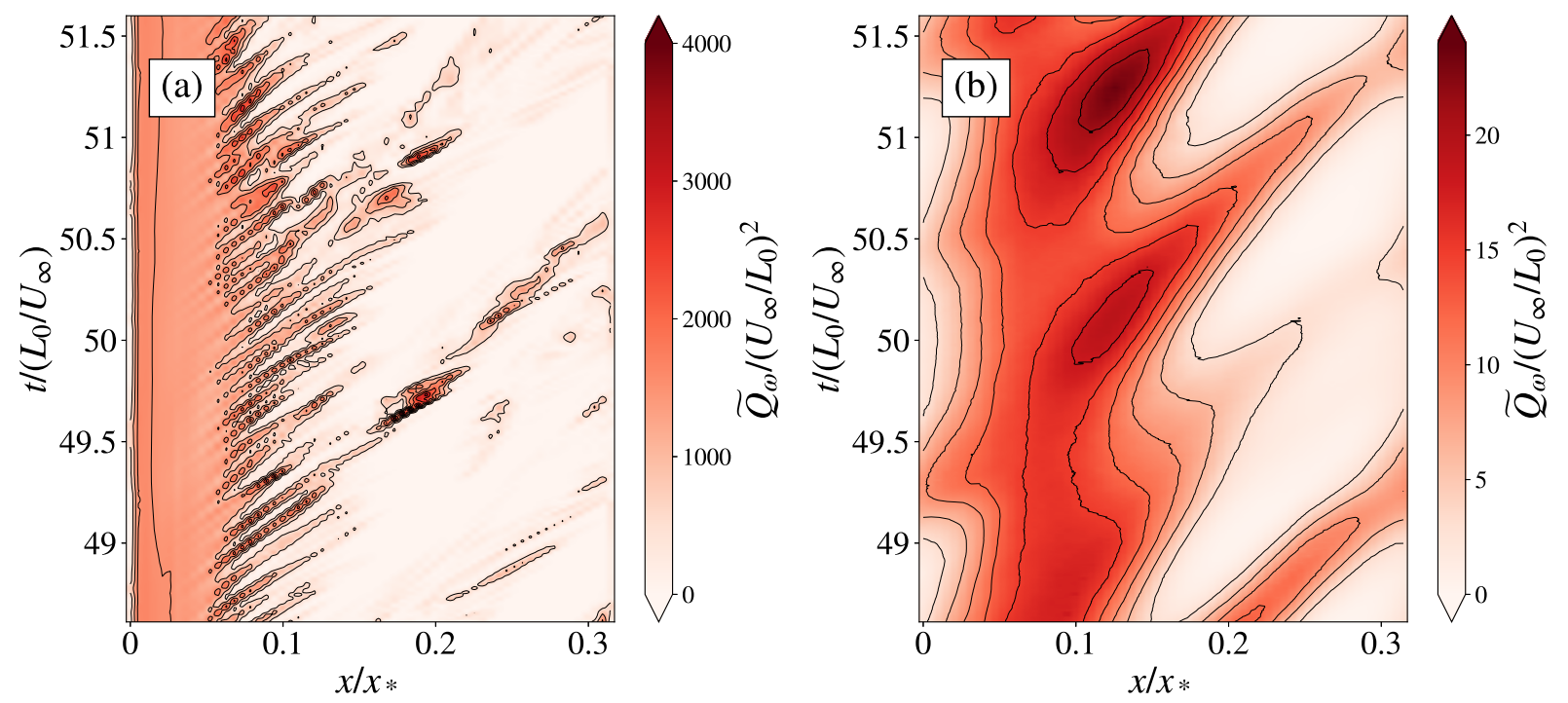

FIG. 8. Space-time evolution of coarse-grained enstrophy $\widetilde{Q}_{\omega}$ along the shear-layer line. The cut-off wavenumbers (11) used are $(\mathrm{a}) k_{c}(2)$ and (b) $k_{c}(0.186)$. We observe the periodic behaviors with the period (a) about $0.1 L_{0} / U_{\infty}$ in the production region at the scale $1 / k_{c}(2)$ and (b) about $L_{0} / U_{\infty}$ in the decay region at the scale $1 / k_{c}(0.186)$.

behaviors of the energy and pressure spectra may be attributed to the non-locality of the pressure field.

\section{Streamwise evolution of coherent vortices}

In Sec. III B, we have demonstrated the streamwise evolutions of energy and pressure spectra along the shear-layer line. In the spectra, we have detected two characteristic frequencies, the vortex-shedding frequency $(\mathrm{St}=0.186)$ and the shear-layer frequency $(2 \lesssim \mathrm{St} \lesssim 3)$, and confirmed their streamwise evolution. It is noticeable in Figs. 5(b,d) that rapid temporal fluctuations are first excited along the shear-layer line, which is the spatial origin of intense temporal fluctuations. The purpose of this subsection is to elucidate a connection between the energy and pressure spectra observed in the previous subsection and several-scale coherent structures in the grid-generated turbulence.

For achieving this, we use the coarse-graining method introduced in Sec. II B. Recall that we need to determine the filter scale $\sigma$ in Eq. (7), or equivalently the cut-off wavenumber $k_{c}$ in Eq. (10). Here, we show evidence for the validity of Eq. (11) for determining $k_{c}$ as a 
function of frequency $f$. Figure 8 shows the space-time diagrams of the enstrophy $\widetilde{Q}_{\omega}$ coarsegrained at two different wavenumbers. The cut-off wavenumbers used in Figs. 8(a) and 8(b) for Gaussian low-pass filtering are $k_{c}(2)$ and $k_{c}(0.186)$, respectively, which correspond to the shear-layer frequency $\mathrm{St}=2$ and the vortex-shedding frequency $\mathrm{St}=0.186$. We can confirm that high-frequency coherent signals are observed in the contour of $\widetilde{Q}_{\omega}$ with $k_{c}(2)$ in the range $0.05 \lesssim x / x_{*} \lesssim 0.1$ [see Fig. 8(a)]. The high-frequency coherent signals are relevant to the shear-layer dynamics because the period of the temporal oscillation is about $0.1 L_{0} / U_{\infty}$, which corresponds to $\mathrm{St}=2$ because $2 U_{\infty} / t_{0} \approx 10 U_{\infty} / L_{0}$. Recall that $L_{0} / t_{0}=5.3$. As the cut-off wavenumber is decreased, such signals become less and less evident as smaller-scale spatial fluctuations are filtered out. As a result, only slowly evolving and large-scale fluctuations are observed in the space-time diagram for the cut-off wavenumber $k_{c}\left(\mathrm{St}=\mathrm{St}_{s}=0.186\right)$ [Fig. 8(b)]. This figure shows that the time scale of the temporal oscillation is about $L_{0} / U_{\infty}$, which corresponds approximately to the non-dimensional frequency St $=f t_{0} / U_{\infty}=0.186$. Hence, temporal and spatial signals are reasonably connected using the low-pass filtering with the cut-off wavenumber (11).

We now compare the isosurfaces [Fig. 9(a)] of the unfiltered second invariant (5) of the velocity gradient tensor with those [Figs. 9(b) and (c)] of filtered ones. The streamwise range in Fig. 9 is $0 \leq x / x_{*} \leq 0.419$ and the two spanwise ranges are $-1 \leq y / L_{0}$ and $z / L_{0}<1$. The isosurfaces visualized in Fig. 9(a) are found to be small-scale tube-like structures and large-scale spatial coherence is not observed in this visualization. Figure 9(a) clearly shows that we need coarse-graining (Sec. II B) of the velocity gradients to capture coherent vortical structures larger than the dissipative scale.

Let us move to the larger-scale coherent vortices observed in Figs. 9(b) and 9(c), which are coarse-grained with the cut-off wavenumbers $k_{c}(2)$ and $k_{c}(0.186)$. Looking at blue vortices in Fig. 9(c), we observe large-scale coherent shedding vortices whose spatial scale is comparable to the scale of the single-square grid. These large-scale structures have shapes of squares. There are smaller square-shaped coherent structures shed by the inner square wall and larger ones shed by the outer square wall, which appear alternately.

We observe in Fig. 9(b) that the (yellow) vortical structures coarse-grained with the cutoff wavenumber $k_{c}(2)$ are parallel to the bars in the near-field region. This verifies that they are due to a shear-layer instability $[46,47,49-52]$. In the downstream region $\left(x / x_{*} \gtrsim 0.15\right)$, however, the orientation of most of the yellow vortices is not parallel to the bar but they 

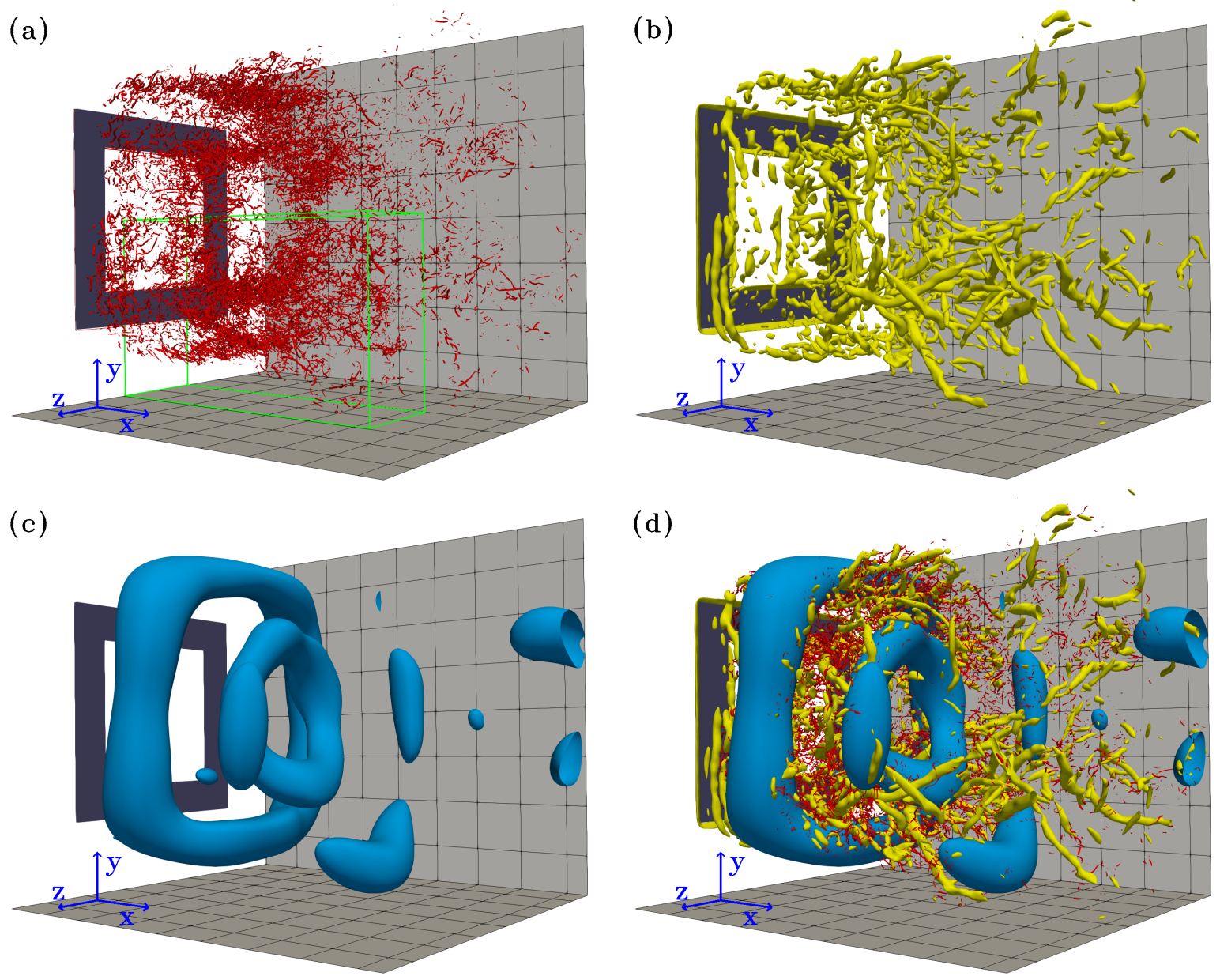

FIG. 9. Visualizations of multi-scale coherent vortices in the computational domain whose streamwise range and spanwise ranges are $0 \leq x / x_{*} \leq 0.42$ and $-1 \leq y / L_{0}, z / L_{0}<1$. They are visualized by isosurfaces of (a) $Q$ and (b,c) $\widetilde{Q}$. The cut-off wavenumbers used for low-pass filtering are $(\mathrm{b}) k_{c}(\mathrm{St}=2.0)$ and $(\mathrm{c}) k_{c}(0.186)$. The values of isosurfaces are (a) $12000\left(U_{\infty} / L_{0}\right)^{2}$, (b) $200\left(U_{\infty} / L_{0}\right)^{2}$, and (c) $1.5\left(U_{\infty} / L_{0}\right)^{2}$. (d) Superimposed visualization. The snapshots are taken at time $t=48.61 L_{0} / U_{\infty}$. The grid width indicates $0.05 x_{*}$.

are in quasi-streamwise direction. Next, we investigate this process in more detail.

Figure 10, which is a magnification of the subdomain of Fig. 9(d) shown by the green box in Fig. 9(a), is a snapshot of coherent structures of different sizes in the near-field region. To further clarify that the yellow vortices are due to shear-flow instability in the region $x / x_{*} \lesssim 0.1$, we show a magnification of Fig. 10 in Fig. 11(a). In this very near-field region the yellow vortical structures are indeed observed as spanwise vortices aligned parallel to the bar. Looking at Fig. 10 again, in the downstream region $x / x_{*} \gtrsim 0.15$, the intermediate (yellow) 


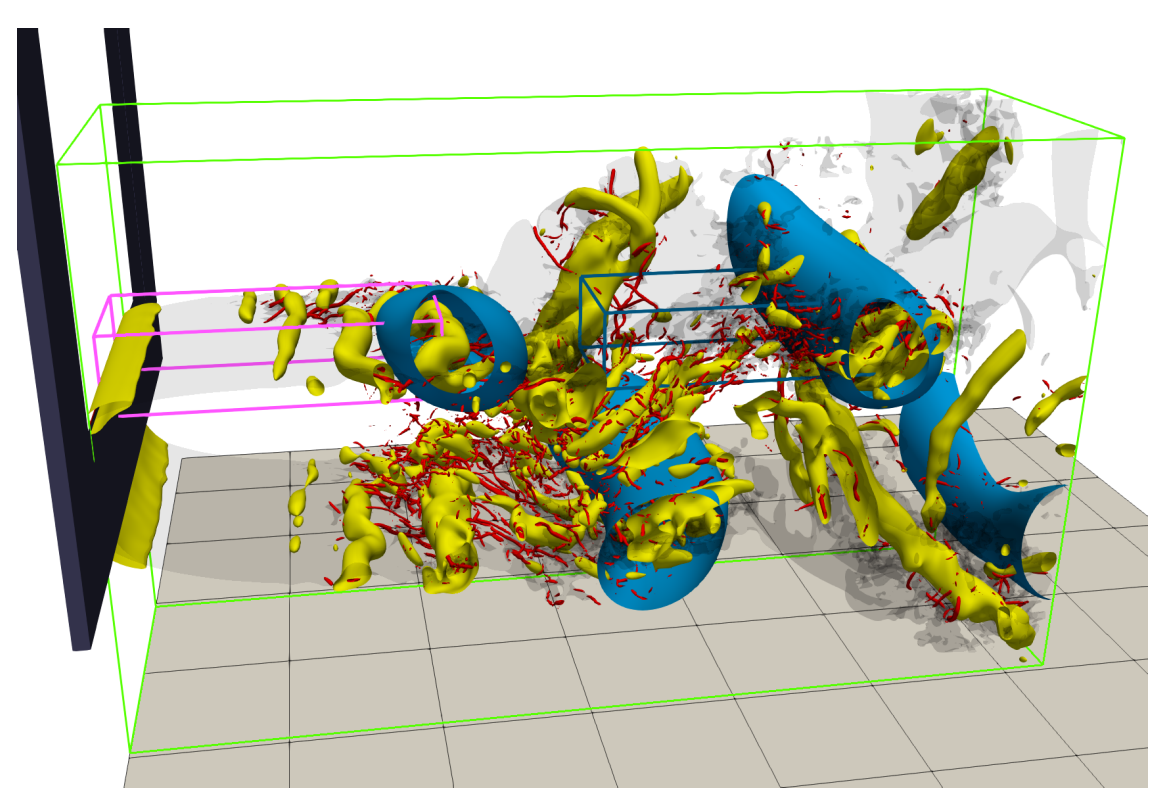

FIG. 10. Snapshot of multi-scale coherent structures in the subdomain $\left(0 \leq x / x_{*} \leq 0.315,-0.75 \leq\right.$ $\left.y / L_{0} \leq 0.25\right)$. Red isosurfaces denote $Q=12000\left(U_{\infty} / L_{0}\right)^{2}$. Yellow isosurfaces denote $\widetilde{Q}=$ $120\left(U_{\infty} / L_{0}\right)^{2}$ with the cut-off wavenumber $k_{c}(2)$. Blue isosurfaces denote $\widetilde{Q}=1.5\left(U_{\infty} / L_{0}\right)^{2}$ with the cut-off wavenumber $k_{c}(0.186)$. Transparent grey isosurface denote $u_{x}=U_{\infty}$. The snapshot is taken at time $t=48.89 L_{0} / U_{\infty}$. The cuboid domains drawn with red and blue lines are used in Figs. 11(a) and 11(b), respectively. The grid width indicates $0.05 x_{*}$.

vortices tend to align with the stretching direction which bridges the counter-rotating large vortices. It is a mechanism which could be a priori linked to a cascading event originating from the largest (shedding) vortices. However, recall that broad continuous energy spectra, even $-5 / 3$ power law spectra, are established well before this. They are in fact established in the region $0.1 \lesssim x / x_{*} \lesssim 0.15$. In this upstream region, a stretching field between a yellow vortex created by the shear-layer instability and a shedding (blue) vortex plays an important role. In fact, we observe in the lower region in Fig. 10 that an anti-clockwise yellow vortex located around $x / x_{*} \approx 0.1$ and an anti-clockwise blue vortex located around $x / x_{*} \approx 0.2$ cooperate to stretch and create intermediate-scale vortices between them. This may be related to the reason why the continuous energy spectrum is established in the region $0.1 \lesssim x / x_{*} \lesssim 0.15$

We now examine how the smallest-scale (red) vortices are created. In the very near field [Fig. 11(a)], the smallest-scale (red) vortices align in the direction perpendicular to the axes 

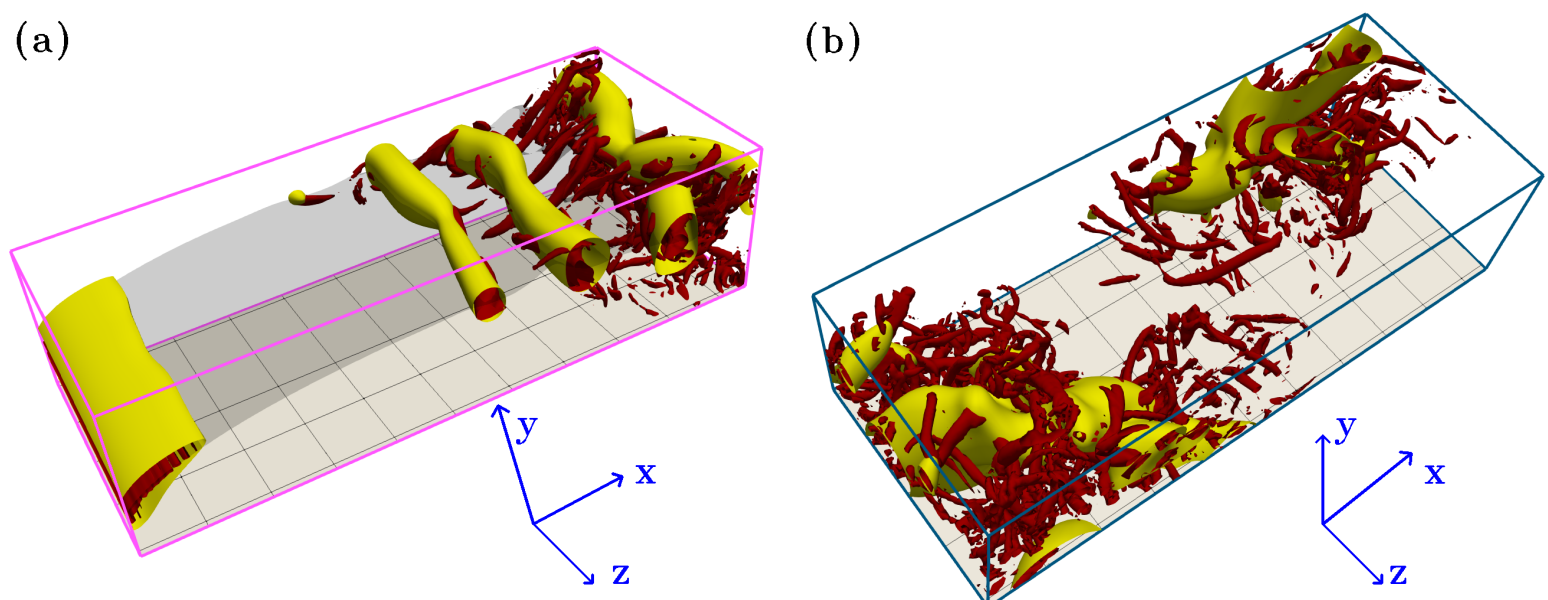

FIG. 11. Snapshot of coherent structures in the cropped domains shown in Fig. 10: (a) the cuboid domain with red lines $(0 \leq x \leq 0.105)$, and (b) the cuboid domain with blue lines $(0.157 \leq x \leq$ 0.262). $t=49.58 L_{0} / U_{\infty}$. (a,b) Red isosurfaces denote $Q=2000\left(U_{\infty} / L_{0}\right)^{2}$. Yellow isosurfaces denote $\widetilde{Q}=120\left(U_{\infty} / L_{0}\right)^{2}$ with the cut-off wavenumber $k_{c}(2)$. (a) Transparent grey isosurface denote $u_{x}=U_{\infty}$. The grid width indicates $0.01 x_{*}$.

of the yellow vortices, which are created by the shear-layer instability. This suggests that the smallest-scale vortices are generated by vortex stretching in the straining fields between intermediate-scale (yellow) spanwise co-rotating vortices.

Figure 11(b) shows the smallest-scale vortices further downstream, specifically the cropped domain with blue lines in Fig. 10, whose streamwise range $\left(0.157 \leq x / x_{*} \leq 0.262\right)$ is within the decay side on the shear-layer line. A large number of clustered small-scale tubular (red) vortices appear in the vicinity of intermediate-scale (yellow) vortices. The orientation of these small-scale vortices is random in the decay side of the shear-layer line, whereas it is strongly anisotropic in the very near-field side of this line [see Fig. 11(a)].

In summary, the development of the energy spectrum is related to the generation process of the coherent vortical structures. In the very near-field region, intermediate (yellow) vortices are first generated due to the shear-layer instability [Fig. 11(a)]. Their spectral signature is the strong spectral peak at $\mathrm{St} \approx 2-3$ observed at $x / x_{*} \approx 0.05$, see Figs. $7(\mathrm{a}, \mathrm{b}, \mathrm{c}, \mathrm{d})$. When the (blue) shedding vortices are established around $x / x_{*} \approx 0.1$, the peak at $\mathrm{St}=0.186$ becomes conspicuous in $E_{x}$ and $E_{y}$ [Figs. $\left.7(\mathrm{a}, \mathrm{b})\right]$. One can expect the interactions be- 
tween these shedding vortices and the shear-layer vortices to excite turbulence fluctuations and vortices at intermediate length-scales, see Fig. 10, so that frequencies in the range $0.186<$ St $\lesssim 2$ acquire energy. Perhaps surprisingly, the energy spectrum appears to tend to a close to $-5 / 3$ power-law scaling as a result of this cooperative process (Fig. 7 ) in the region $0.1 \lesssim x / x_{*} \lesssim 0.15$ on the shear-layer line. Further downstream on this line, where the near to $-5 / 3$ power law of the energy spectra persists, the blue large-scale vortices remain present. This is consistent with the persistence of peak at St $=0.186$ which remains clearly well-defined in the spectra (Fig. 7).

\section{Streamwise evolution of scale-by-scale energy transfer}

The presence of large-scale coherent structures can be expected to interfere with interscale energy transfer and may be a key ingredient leading to non-equilibrium, i.e. unsteadiness in time and non-uniformity across scales of inter-scale transfer rates [9, 12, 53-56]. Having demonstrated the streamwise evolution of spectra (Sec. III B) and characteristic coherent vortical structures (Sec. III C), we now discuss inter-scale energy transfers by evaluating the streamwise evolution of the scale-by-scale turbulent energy flux along the shear-layer line. A reference situation is homogeneous stationary turbulence where the spherically averaged inter-scale flux is balanced by the turbulence dissipation rate [9, 45, 57-59], as

$$
\int \widehat{\boldsymbol{r}} \cdot\left\langle\frac{\delta \boldsymbol{u}}{r}|\delta \boldsymbol{u}|^{2}\right\rangle \mathrm{d} \Omega \approx-\frac{16 \pi}{3}\langle\epsilon\rangle
$$

where $\delta \boldsymbol{u}\left(\boldsymbol{x}, \boldsymbol{r}_{d}, t\right)=\boldsymbol{u}(\boldsymbol{\xi}, t)-\boldsymbol{u}\left(\boldsymbol{\xi}^{\prime}, t\right)$ is the two-point velocity difference, $\boldsymbol{x}=\left(\boldsymbol{\xi}+\boldsymbol{\xi}^{\prime}\right) / 2$ is the centroid, $\boldsymbol{r}_{d}=\boldsymbol{\xi}-\boldsymbol{\xi}^{\prime}$ is the separation vector, $\boldsymbol{r}=\boldsymbol{r}_{d} / 2, \widehat{\boldsymbol{r}}=\boldsymbol{r} / r$ and $\mathrm{d} \Omega$ is the differential of the solid angle in the $\boldsymbol{r}$-space. The minus sign on the right-hand side indicates a forward cascade, from large to small scales. If turbulent velocity field is locally isotropic such that $\widehat{\boldsymbol{r}} \cdot\left\langle\delta \boldsymbol{u}|\delta \boldsymbol{u}|^{2}\right\rangle$ is the same irrespective of the orientation of the unit vector $\widehat{\boldsymbol{r}}$, then Eq. (16) leads to the $4 / 3$ law $[9,57,59-61]$. This law is equivalent to the $4 / 5$ law for the third-order longitudinal velocity structure function.

We define and compute the normalized spherically averaged energy flux $\Pi^{*}(\boldsymbol{x}, r)$

$$
\Pi^{*}\left(\boldsymbol{x}, r_{d}\right)=\left\langle\int \widehat{\boldsymbol{r}} \cdot \frac{\delta \boldsymbol{u}}{r}|\delta \boldsymbol{u}|^{2} \mathrm{~d} \Omega\right\rangle /\left(-\frac{16 \pi}{3}\langle\epsilon\rangle\right),
$$

where $\langle\epsilon\rangle$ is computed at the centroid $\boldsymbol{x}$. If the energy flux balances over scales, a constant scale range emerges in the distribution of $\Pi^{*}\left(\boldsymbol{x}, r_{d}\right)$ against $r_{d}$. Figure 12 shows the scale 


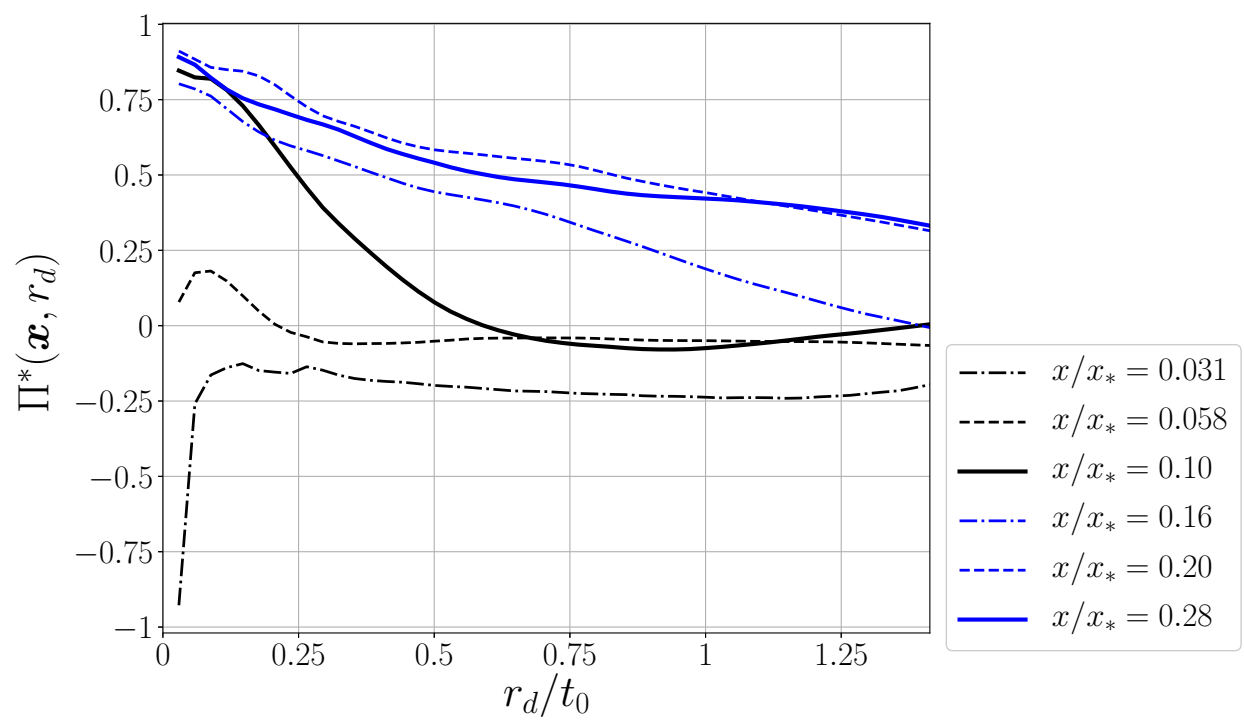

FIG. 12. Scale distribution of normalized spherically-averaged energy flux $\Pi^{*}\left(\boldsymbol{x}, r_{d}\right)$ in physical space. $\boldsymbol{x}$ is chosen at six different locations on the shear-layer line whose streamwise location is $x / x_{*}=0.031,0.058,0.10,0.16,0.20,0.28$.

distribution of $\Pi^{*}\left(\boldsymbol{x}, r_{d}\right)$ at six different centroids $\boldsymbol{x}$ on the shear-layer line. The streamwise locations of these centroids are $x / x_{*}=0.031,0.058,0.1,0.16,0.2,0.28$. With the use of Eq. (11), the spatial scale $r_{d}=0.5 t_{0}$ relates to the shear-layer instability frequency $\mathrm{St}=2$. We find that the distribution of $\Pi^{*}\left(\boldsymbol{x}, r_{d}\right)$ against $r_{d}$ varies significantly with centroid position. The three black-colored curves correspond to the production region $(0 \leq$ $\left.x / x_{*} \lesssim 0.15\right)$ where the spectra do not exhibit Kolmogorov-like scalings yet, whereas the three blue-colored curves are obtained in the decay region $\left(0.15 \lesssim x / x_{*}\right)$. Very near the grid bar $\left(x / x_{*}=0.031\right), \Pi^{*}\left(\boldsymbol{x}, r_{d}\right)$ is negative over the entire range of $r_{d}$, suggesting the energy flowing from small to large scales. $\Pi^{*}\left(\boldsymbol{x}, r_{d}\right)$ becomes clearly positive over all scales $r_{d}$ up to at least $1.25 t_{0}$ once the decay region is reached. From $x / x_{*}=0.10$ to 0.16 , where we identified the cooperative action between the shear-layer and the shedding vortices, the power-law exponent of the energy spectra transients from near $-7 / 3$ to near $-5 / 3$ [see Fig. $7(\mathrm{a}, \mathrm{b}, \mathrm{c})]$. The behaviour of $\Pi^{*}\left(\boldsymbol{x}, r_{d}\right)$ in this region evolves quickly and involves both positive and negative values of $\Pi^{*}\left(\boldsymbol{x}, r_{d}\right)$. The end of the production region and start of the decay region around $x / x_{*}=0.16$ coincides with the appearance of near $-5 / 3$ spectra. It is from that point and moving downstream along the shear-layer line that the sign of $\Pi^{*}\left(\boldsymbol{x}, r_{d}\right)$ 
is uniformly positive (at least in the range $r_{d} / t_{0} \lesssim 1.25$, i.e. St $\gtrsim 0.8$ ) suggesting that fluctuating kinetic energy is transferred, on average, from larger to smaller scales. However $\Pi^{*}\left(\boldsymbol{x}, r_{d}\right)$ is neither equal to 1 nor in fact constant with varying $r_{d}$ in the decay region (see Fig. 12), which is what one would have expected in homogeneous stationary turbulence in the presence of $-5 / 3$ energy spectra. Of course, the present flow field is not statistically homogeneous and one should not necessarily expect Kolmogorov equilibrium scalings, but it is noteworthy that $-5 / 3$ energy spectra appear without constant $\Pi^{*}\left(\boldsymbol{x}, r_{d}\right)$. Moving further downstream on the shear-layer line, $\Pi^{*}\left(\boldsymbol{x}, r_{d}\right)$ shows a tendency towards a broad range constancy but does not reach it till the most downstream position in Fig. 12, which is $x / x_{*}=0.28$. However, the pressure spectrum which varies with downstream position on the shear-layer line [see Fig. 7 (d)] achieves its Kolmogorov-like $-7 / 3$ shape at $x / x_{*}=0.28$, i.e. before any constancy of $\Pi^{*}\left(\boldsymbol{x}, r_{d}\right)$ might be achieved. The results of Ref. [12] and Ref. [9] suggest that $\Pi^{*}\left(\boldsymbol{x}, r_{d}\right)$ might well achieve constancy further downstream, perhaps even a constant value close to -1 , but in a region which remains highly inhomogeneous.

\section{CONCLUSIONS}

Near $-5 / 3$ and $-7 / 3$ power-law scalings of the energy and pressure frequency spectra are rapidly established in the near-field region behind bars of a grid obstructing a uniform laminar stream. The flow generated by the bars is statistically highly inhomogeneous, yet the exponents of the spectra are reminiscent of Kolmogorov's theory [1] predictions. In this study, by virtue of long-time DNS integrations, we have shed some light on the origin of these spectra.

We have found peaks at two characteristic frequencies in both the energy and the pressure spectra: a vortex-shedding frequency $(\mathrm{St}=0.186)$ and a shear-layer frequency $(\mathrm{St} \approx 2)$. These frequencies appear immediately in different spectra, but the shear-layer's spectral signature is very soon replaced by a broad spectrum on both sides of that frequency. The spectra evolve further by filling the gap between the vortex shedding and the shear-layer frequencies, eventually leading to a near $-5 / 3$ power-law energy spectra at the point on the shear-layer line where the turbulence intensity reaches a maximum. Interestingly, the

pressure spectrum does not evolve in the same way, but reaches a $-7 / 3$ power-law shape significantly further downstream [Fig. 7(d)]. 
We discussed the spatial development of these spectra in terms of turbulent coherent structures of various sizes, obtained by using Gaussian low-pass filtering (8) of instantaneous turbulent flow fields. Concretely, in this very near field turbulence, intermediatescale vortices (the yellow isosurfaces in Figs. 9-11) emerge in a slightly downstream region $\left(x / x_{*} \approx 0.05\right)$ because of the shear-layer instability, while large shedding vortices (the blue isosurfaces in Figs. 9 and 10) are fully established in a downstream region $\left(x / x_{*} \approx 0.1\right)$. These two different coherent vortices at two different length scales have a large gap in their time scales and interact cooperatively to generate intermediate-scale vortices in the region $0.1 \lesssim x / x_{*} \lesssim 0.15$ (Fig. 10), thereby rapidly leading to energy spectra proportional to, approximately, $f^{-5 / 3}$ in a broad range of frequencies (Fig. 7). We emphasize that this process is different from the conventional concept of an energy cascade with constant scale-by-scale energy flux (Fig. 12).

Although we observe smaller-scale vortices (the red isosurfaces in Figs. 9-11) around the intermediate-scale yellow vortices, which are relevant to frequencies higher than St $>3$ in the spectra of Fig. 7, this high-frequency region does not appear to be so important for the establishment of the near $-5 / 3$ power-law scalings at the Reynolds number examined here. The pressure spectrum established a near $-7 / 3$ power-law scaling significantly further downstream, though also in the near-field very inhomogeneous turbulence. At the downstream point where this $-7 / 3$ power-law scaling finally appears, the scale-by-scale energy flux which evolves significantly along the shear-layer line is still not constant over a broad range of scales (Fig. 12). Further future investigations of the differences in the way energy and pressure spectra develop (Fig. 7) are needed for a better understanding of turbulent inter-scale transfers and their potentially important relations with spatial energy transfers.

\section{ACKNOWLEDGMENTS}

TY and JCV were supported by ERC Advanced Grant 320560. SG was supported by the JSPS Grant-in-Aid for Scientific Research (16H04268).

[1] A. N. Kolmogorov, "The local structure of turbulence in incompressible viscous fluid for very large Reynolds numbers," Dokl. Akad. Nauk SSSR 30, 301-305 (1941). 
[2] W. K. George, P. D. Beuther, and R. E. A. Arndt, "Pressure spectra in turbulent free shear flows," J. Fluid Mech. 148, 155-191 (1984).

[3] Y. Tsuji and T. Ishihara, "Similarity scaling of pressure fluctuation in turbulence," Phys. Rev. E 68, 026309 (2003).

[4] O. Terashima, Y. Sakai, and K. Nagata, "Simultaneous measurement of velocity and pressure in a plane jet," Exp. Fluids 53, 1149-1164 (2012).

[5] T. Gotoh and D. Fukayama, "Pressure spectrum in homogeneous turbulence," Phys. Rev. Lett. 86, 3775-3778 (2001).

[6] T. Yasuda, S. Goto, and G. Kawahara, "Quasi-cyclic evolution of turbulence driven by a steady force in a periodic cube," Fluid Dyn. Res. 46, 061413 (2014).

[7] S. Goto, Y. Saito, and G. Kawahara, "Hierarchy of antiparallel vortex tubes in spatially periodic turbulence at high Reynolds numbers," Phys. Rev. Fluids 2, 064603 (2017).

[8] S. Laizet, J. C. Vassilicos, and C. Cambon, "Interscale energy transfer in decaying turbulence and vorticity-strain rate dynamics in grid-generated turbulence," Fluid Dyn. Res. 45, 061408 (2013).

[9] R. Gomes-Fernandes, B. Ganapathisubramani, and J. C. Vassilicos, "The energy cascade in near-field non-homogeneous non-isotropic turbulence," J. Fluid Mech. 771, 676-705 (2015).

[10] I. Paul, G. Papadakis, and J. C. Vassilicos, "Genesis and evolution of velocity gradients in near-field spatially developing turbulence," J. Fluid Mech. 815, 295-332 (2017).

[11] J. Nedić, J. C. Vassilicos, and B. Ganapathisubramani, "Axisymmetric turbulent wakes with new nonequilibrium similarity scalings," Phys. Rev. Lett. 111, 144503 (2013).

[12] F. Alves Portela, G. Papadakis, and J. C. Vassilicos, "The turbulence cascade in the near wake of a square prism," J. Fluid Mech. 825, 315-352 (2017).

[13] Y. Zhou, K. Nagata, Y. Sakai, H. Suzuki, Y. Ito, O. Terashima, and T. Hayase, "Development of turbulence behind the single square grid," Phys. Fluids 26, 045102 (2014).

[14] Y. Zhou, K. Nagata, Y. Sakai, H. Suzuki, Y. Ito, O. Terashima, and T. Hayase, "Relevance of turbulence behind the single square grid to turbulence generated by regular- and multiscalegrids," Phys. Fluids 26, 075105 (2014).

[15] S. Laizet, J. Nedić, and J. C. Vassilicos, "The spatial orgin of $-5 / 3$ spectra in grid-generated turbulence," Phys. Fluids 27, 065115 (2015). 
[16] G. Melina, P. J. K. Bruce, and J. C. Vassilicos, "Vortex shedding effects in grid-generated turbulence," Phys. Rev. Fluids 1, 044402 (2016).

[17] Y. Zhou, K. Nagata, Y. Sakai, Y. Ito, and T. Hayase, "Spatial evolution of the helical behavior and the $2 / 3$ power-law in single-square-grid-generated turbulence," Fluid Dyn. Res. 48, 021404 (2016).

[18] S. Goto, "A physical mechanism of the energy cascade in homogeneous isotropic turbulence," J. Fluid Mech. 605, 355-366 (2008).

[19] T. Leung, N. Swaminathan, and P. A. Davidson, "Geometry and interaction of structures in homogeneous isotropic turbulence," J. Fluid Mech. 710, 453-481 (2012).

[20] S. Goto, "Coherent structures and energy cascade in homogeneous turbulence," Prog. Theor. Phys. Supp. 195, 139-156 (2012).

[21] J. I. Cardesa, A. Vela-Martín, and J. Jiménez, "The turbulent cascade in five dimensions," Science 357, 782-784 (2017).

[22] Y. Motoori and S. Goto, "Generation mechanism of a hierarchy of vortices in a turbulent boundary layer," J. Fluid Mech. 865, 1085-1109 (2019).

[23] A. Lozano-Durán, M. Holzner, and J. Jiménez, "Multiscale analysis of the topological invariants in the logarithmic region of turbulent channels at a friction Reynolds number of 932," J. Fluid Mech. 803, 356-394 (2016).

[24] J. Lee, H. J. Sung, and T. A. Zaki, "Signature of large-scale motions on turbulent/nonturbulent interface in boundary layers," J. Fluid Mech. 819, 165-187 (2017).

[25] S. Laizet and E. Lamballais, "High-order compact schemes for incompressible flows: A simple and efficient method with quasi-spectral accuracy," J. Comput. Phys. 228, 5989-6015 (2009).

[26] S. Laizet and N. Li, "Incompact3d: A powerful tool to tackle turbulence problems with up to $\mathrm{O}\left(10^{5}\right)$ computational cores," Int. J. Numer. Meth. Fluids 67, 1735-1757 (2011).

[27] S. K. Lele, "Compact finite difference schemes with spectral-like resolution," J. Comput. Phys. 103, 16-42 (1992).

[28] P. Parnaudeau, J. Carlier, D. Heitz, and E. Lamballais, "Experimental and numerical studies of the flow over a circular cylinder at Reynolds number 3900," Phys. Fluids 20, 085101 (2008).

[29] S. Laizet, J. Nedić, and J. C. Vassilicos, "Influence of the spatial resolution on fine-scale features in DNS of turbulence generated by a single square grid," Int. J. Comput. Fluid Dyn. 29, 286-302 (2015). 
[30] M. S. Chong, A. E. Perry, and B. J. Cantwell, "A general classification of three-dimensional flow fields," Phys. Fluids A 2, 765-777 (1990).

[31] N. Mazellier and J. C. Vassilicos, "Turbulence without Richardson-Kolmogorov cascade," Phys. Fluids 22, 075101 (2010).

[32] R. Gomes-Fernandes, B. Ganapathisubramani, and J. C. Vassilicos, "Particle image velocimetry study of fractal-generated turbulence," J. Fluid Mech. 711, 306-336 (2012).

[33] U. Fey, M. König, and H. Eckelmann, "A new Strouhal-Reynolds-number relationship for the circular cylinder in the range $47<R e<2 \times 10^{5}$," Phys. Fluids 10, 1547-1549 (1998).

[34] K. Yamamoto and I. Hosokawa, "A decaying isotropic turbulence pursued by the spectral method," J. Phys. Soc. Jpn. 57, 1532-1535 (1988).

[35] J. Jiménez, A. A. Wray, P. G. Saffman, and R. S. Rogallo, "The structure of intense vorticity in isotropic turbulence," J. Fluid Mech. 255, 65-90 (1993).

[36] J. Soria, R. Sondergaard, B. J. Cantwell, M. S. Chong, and A. E. Perry, "A study of the fine-scale motions of incompressible time-developing mixing layers," Phys. Fluids 6, 871-884 (1994).

[37] H. M. Blackburn, N. N. Mansour, and B. J. Cantwell, "Topology of fine-scale motions in turbulent channel flow," J. Fluid Mech. 310, 269-292 (1996).

[38] J. M. Chacín, B. J. Cantwell, and S. J. Kline, "Study of turbulent boundary layer structure using the invariants of the velocity gradient tensor," Exp. Therm. Fluid Sci. 13, 308-317 (1996).

[39] A. Tsinober, An Informal Introduction to Turbulence (Springer Netherlands, 2014).

[40] R. Gomes-Fernandes, B. Ganapathisubramani, and J. C. Vassilicos, "Evolution of the velocitygradient tensor in a spatially developing turbulent flow," J. Fluid Mech. 756, 252-292 (2014).

[41] O. R. H. Buxton, M. Breda, and X. Chen, "Invariants of the velocity-gradient tensor in a spatially developing inhomogeneous turbulent flow," J. Fluid Mech. 817, 1-20 (2017).

[42] F. H. Champagne, "The fine-scale structure of the turbulent velocity field," J. Fluid Mech. 86, 67-108 (1978).

[43] U. Frisch, Turbulence: The Legacy of A. N. Kolmogorov (Cambridge Univ. Press, 1995).

[44] D. Hurst and J. C. Vassilicos, "Scalings and decay of fractal-generated turbulence," Phys. Fluids 19, 035103 (2007).

[45] J. C. Vassilicos, "Dissipation in turbulent flows," Annu. Rev. Fluid Mech. 47, 95-114 (2015). 
[46] M. S. Bloor, "The transition to turbulence in the wake of a circular cylinder," J. Fluid Mech. 19, 290-304 (1964).

[47] C. H. K. Williamson, "Vortex dynamics in the cylinder wake," Annu. Rev. Fluid Mech. 28, 477-539 (1996).

[48] A. Prasad and C. H. K. Williamson, "The instability of the shear layer separating from a bluff body," J. Fluid Mech. 333, 375-402 (1997).

[49] J. Jiménez, R. Martinez-Val, and M. Rebollo, "On the origin and evolution of three dimensional effects in the mixing layer," Final Technical Report (1979).

[50] C.-M. Ho and P. Huerre, "Perturbed free shear layers," Annu. Rev. Fluid Mech. 16, 365-424 (1984).

[51] T. Watanabe, J. J. Riley, K. Nagata, R. Onishi, and K. Matsuda, "A localized turbulent mixing layer in a uniformly stratified environment," J. Fluid Mech. 849, 245-276 (2018).

[52] K. Takamure, Y. Ito, Y. Sakai, K. Iwano, and T. Hayase, "Momentum transport process in the quasi self-similar region of free shear mixing layer," Phys. Fluids 30, 015109 (2018).

[53] S. Goto and J. C. Vassilicos, "Unsteady turbulence cascades," Phys. Rev. E 94, 053108 (2016).

[54] P. C. Valente and J. C. Vassilicos, "The energy cascade in grid-generated non-equilibrium decaying turbulence," Phys. Fluids 27, 045103 (2015).

[55] T. Yasuda and J. C. Vassilicos, "Spatio-temporal intermittency of the turbulent energy cascade," J. Fluid Mech. 853, 235-252 (2018).

[56] T. Yasuda, G. Kawahara, L. van Veen, and S. Kida, "A vortex interaction mechanism for generating energy and enstrophy fluctuations in high-symmetric turbulence," J. Fluid Mech. 874, 639-679 (2019).

[57] Q. Nie and S. Tanveer, "A note on third-order structure functions in turbulence," Proc. Roy. Soc. Lond. A 455, 1615-1635 (1999).

[58] J. Duchon and R. Robert, "Inertial energy dissipation for weak solutions of incompressible Euler and Navier-Stokes equations," Nonlinearity 13, 249-255 (2000).

[59] G. L. Eyink, "Local 4/5-law and energy dissipation anomaly in turbulence," Nonlinearity 16, 137-145 (2003).

[60] R. J. Hill, "Exact second-order structure-function relationships," J. Fluid Mech. 468, 317-326 (2002). 
[61] L. Danaila, J. F. Krawczynski, F Thiesset, and B Renou, "Yaglom-like equation in axisymmetric anisotropic turbulence," Physica D 241, 216-223 (2012). 